\title{
Intraperitoneal and Systemic Chemotherapy for Patients with Gastric Cancer with Peritoneal Metastasis: A Phase II Trial
}

\author{
MASAYUKI SHINKAI $^{1}$, MOTOHIRO IMANO $^{1}$, YASUTAKA CHIBA $^{2}$, YOKO HIRAKI $^{1}$, \\ HIROAKI KATO ${ }^{1}$, MITSURU IWAMA ${ }^{1}$, OSAMU SHIRAISHI ${ }^{1}$, ATSUSHI YASUDA ${ }^{1}$, \\ MASAHIRO TSUBAKI ${ }^{3}$, SHOZO NISHIDA $^{3}$, YUTAKA KIMURA ${ }^{1}$ and TAKUSHI YASUDA ${ }^{1}$ \\ ${ }^{1}$ Department of Surgery, Faculty of Medicine, and ${ }^{3}$ Division of Pharmacotherapy, \\ Faculty of Pharmacy, Kindai University, Osaka, Japan; \\ ${ }^{2}$ Clinical Research Center, Kindai University Hospital, Osaka, Japan
}

\begin{abstract}
Aim: To conduct a phase II study of single intraperitoneal (i.p.) administration of paclitaxel followed by paclitaxel, cisplatin, and S-1 (PCS) chemotherapy for patients with gastric cancer with peritoneal metastasis (PM). Patients and Methods: Staging laparotomy was performed to confirm PM. Initially, patients received i.p. paclitaxel. Beginning 7 days later, PCS was given every 3 weeks followed by second-look laparoscopy. Primary and secondary endpoints were the overall survival (OS) rate, and response rate and patient safety, respectively. Results: Seventeen patients were enrolled. The overall response rate was $70.5 \%$ (12/17). Grade $3 / 4$ toxic effects included neutropenia and leukopenia. After chemotherapy, PM disappearance was confirmed in 11 patients. Gastrectomy was eventually performed in 11 patients. The 1-year OS rate was $82.4 \%$ and the median survival time was 23.9 months considering the overall cohort. Conclusion: Combination chemotherapy with i.p. paclitaxel and PCS is well tolerated and effective in patients with gastric cancer with PM.
\end{abstract}

Gastric cancer (GC) is a life-threatening disease worldwide. Although recent advances in the treatment of GC have improved clinical outcome (1), patients with GC with peritoneal metastasis (PM) still have a poor overall prognosis (2). Recently, numerous modalities have been tried for treating PM, including aggressive surgery, intraperitoneal chemotherapy (IPC), and hyperthermia, but none has provided a satisfactory clinical outcome (3-5). Consequently, there is no standard treatment for patients with PM of GC.

Correspondence to: Motohiro Imano, MD, Ph.D., Department of Surgery, Kindai University Faculty of Medicine, 377-2 Ohnohigashi, Osaka-sayama, Osaka 587-8511, Japan. Tel: +81 723660221, Fax:+81 723677771, e-mail: imano@med.kindai.ac.jp

Key Words: Gastric cancer, intraperitoneal chemotherapy, S-1, cisplatin, paclitaxel, peritoneal metastasis.
S-1 and paclitaxel have a high transition rate in the peritoneal cavity and a high efficacy against diffuse-type adenocarcinoma, which can easily disseminate $(6,7)$. Therefore, S-1 and paclitaxel may be a suitable systemic chemotherapy against PM. In addition, in patients with advanced or recurrent GC, previous trials involving combination chemotherapy with $\mathrm{S}-1$ and intravenous paclitaxel have reported the good safety and efficacy for measurable lesions $(8,9)$.

Paclitaxel has another advantage in the treatment of PM. When administered intraperitoneally, it exhibits delayed clearance from the peritoneal cavity because of its high molecular weight and bulky structure. In a previous study, we demonstrated the possible effectiveness of paclitaxel in IPC (10). The advantage of IPC exposure is best expressed as the achievement of maximum concentration and area under the curve for the ratio of the drug between the peritoneal cavity and peripheral blood (10). Our previous study showed that the average maximum concentration/area under the curve ratio for paclitaxel was 1,065:1 (10).

To confirm the clinical effects of IPC using paclitaxel, we developed a new chemotherapeutic regimen for treating PM from GCs that involves the addition of a single intraperitoneal (i.p.) administration of paclitaxel followed by systemic S-1 and paclitaxel. In a phase II study, we reported that the median survival time of patients with PM treated with paclitaxel followed by systemic S- 1 and paclitaxel was 21.3 months, with an overall response rate of $65.7 \%$ (11). Although our data seemed superior to those of previous studies, we believe that new treatment is always needed to further improve the prognosis for patients with PM of GC.

Cisplatin is another key chemotherapeutic agent used to treat GC. A recent randomized controlled trial showed that combination chemotherapy with cisplatin and S-1 offered a survival benefit over S-1 alone to patients with advanced GC (12). Even more recently, other studies reported that intravenous administration of paclitaxel and cisplatin, and 
oral administration of S-1 (PCS regimen) had a good efficacy with a favorable response rate and median overall survival (OS) in patients with advanced GC, especially those with PM (13-15).

According to these results, this systemic triple-regimen (PCS) chemotherapy in addition to IPC potentially appeared to contribute to improving the prognosis of GC patients with PM.

Therefore, we conducted a phase II study to evaluate the safety and efficacy of this combination chemotherapy including a single i.p. paclitaxel dose followed by the intravenous PCS triplet regimen for patients with PM of GC.

\section{Patients and Methods}

Study design and patients. This study was performed as a prospective, single-center, clinical phase II study. The eligibility criteria for entry into this study were as follows: Histologically proven adenocarcinoma of the stomach; the presence of PM confirmed by staging laparoscopy; absence of non-curative factors (e.g. distant metastasis to the liver, lung, or lymph nodes), except for PM; no prior treatment including surgery, chemotherapy, or irradiation; no active bleeding; sufficient oral intake; Eastern Cooperative Oncology Group performance status score of 0 or 1; age $<75$ years; adequate bone marrow function (white blood cell count $\geq 3500 / \mu \mathrm{l}$, granulocyte count $\geq 1,500 / \mu \mathrm{l}$, platelet count $\geq 100,000 / \mu \mathrm{l}$, hemoglobin $\geq 9.0 \mathrm{~g} / \mathrm{dl}$ ); adequate renal function (serum creatinine $\leq 1.5 \mathrm{mg} / \mathrm{dl}$ ); adequate liver function (total bilirubin $\leq 1.5$ $\mathrm{mg} / \mathrm{dl}$, aspartate transaminase (AST)/alanine transaminase (ALT)/ alkaline phosphatase $\leq 2.5 \times$ upper limit of normal).

In accordance with the ethical standards of the committee responsible for human experimentation and with the Helsinki Declaration of 1975, as revised in 2008 (16), written informed consent was obtained from all patients at the time of the first staging laparoscopy.

Treatment strategy and end points. Firstly, staging laparoscopy was performed under general anesthesia in patients with GC and serosainvading tumors. Then, among those with PM, i.p. and systemic chemotherapy were administered. Paclitaxel $\left(60 \mathrm{mg} / \mathrm{m}^{2}\right.$ i.p. $)$ was administered during the staging laparoscopy, followed by systemic chemotherapy that started within 1 week after the laparoscopy (11). Systemic chemotherapy consisted of a 21-day cycle of intravenous (i.v.) paclitaxel $\left(80 \mathrm{mg} / \mathrm{m}^{2}\right)$ over $1 \mathrm{~h}$ and cisplatin $\left(30 \mathrm{mg} / \mathrm{m}^{2}\right.$ i.v. $)$ over $1 \mathrm{~h}$ on days 1 and 8 . In addition, S-1 was administered orally $\left(80 \mathrm{mg} / \mathrm{m}^{2} /\right.$ day) on days $1-14$ followed by 7 days of rest (15). Treatment was continued every 3 weeks for eight cycles unless there was disease progression, the patient refused the treatment, or unacceptable toxicity occurred.

After chemotherapy, second-look laparoscopy was performed in all patients (except those with disease progression). This step was followed by gastrectomy with D2 lymph node dissection in patients free of macroscopic GC-derived PM. Patients who underwent surgery then received adjuvant chemotherapy using weekly paclitaxel and S-1 (17). The primary endpoint of this clinical phase II study was OS. Secondary endpoints were the response rate and patient safety.

Evaluation of disease. After i.p. and systemic chemotherapy, all patients were evaluated for their clinical response and any drugrelated toxicity. In patients with a target lesion, the therapeutic
Table I. Patient characteristics and tumor response ( $n=17)$.

\begin{tabular}{|c|c|}
\hline Characteristic & Number \\
\hline \multicolumn{2}{|l|}{ Age, years } \\
\hline Median (range) & $60(39-71)$ \\
\hline \multicolumn{2}{|l|}{ Gender, $\mathrm{n}$} \\
\hline Male & 10 \\
\hline Female & 7 \\
\hline \multicolumn{2}{|c|}{ ECOG performance status, $n$} \\
\hline 0 & 17 \\
\hline 1 & 0 \\
\hline \multicolumn{2}{|l|}{ Macroscopic findings, $\mathrm{n}$} \\
\hline Type 1 & 0 \\
\hline Type 2 & 1 \\
\hline Type 3 & 6 \\
\hline Type 4 & 10 \\
\hline \multicolumn{2}{|l|}{ Histological subtype, $\mathrm{n}$} \\
\hline Differentiated & 6 \\
\hline Undifferentiated & 11 \\
\hline \multicolumn{2}{|l|}{ Clinical T-stage, $\mathrm{n}$} \\
\hline $\mathrm{T} 4 \mathrm{a}$ & 16 \\
\hline $\mathrm{T} 4 \mathrm{~b}$ & 1 \\
\hline \multicolumn{2}{|c|}{ Peritoneal lavage cytology, $\mathrm{n}$} \\
\hline CYO & 2 \\
\hline CY1 & 15 \\
\hline \multicolumn{2}{|c|}{ Peritoneal dissemination, $\mathrm{n}$} \\
\hline No & 0 \\
\hline Yes & 17 \\
\hline \multicolumn{2}{|l|}{ Tumor response, $\mathrm{n}$} \\
\hline \multicolumn{2}{|l|}{ RECIST guideline $(n=5)$} \\
\hline Complete response & 0 \\
\hline Partial response & 4 \\
\hline Stable disease & 1 \\
\hline Progressive disease & 0 \\
\hline \multicolumn{2}{|l|}{ Wall thickness $(n=12)$} \\
\hline Over $30 \%$ decrease & 8 \\
\hline No change & 2 \\
\hline Increase & 2 \\
\hline
\end{tabular}

ECOG: Eastern Cooperative Oncology Group, CY0: peritoneal cytology negative for carcinoma cells, CY1: peritoneal cytology positive for carcinoma cells.

effects were evaluated after two, five, and eight courses of treatment and the results were classified based on the Response Evaluation Criteria in Solid Tumors (RECIST) guidelines (18).

For patients who had no target lesion, the antitumor effects were evaluated based on the wall thickness of the primary tumor by means of multi-detector computed tomography using the air-filling technique. The area in the stomach where the wall thickness was measured corresponded to the area with a biopsy-proven tumor mass. A patient was considered a responder when there was a tumor response or a $30 \%$ improvement in wall thickness on one transverse, coronal, or sagittal image (11). Toxicity was graded according to National Cancer Institute Common Toxicity Criteria Version 4.0 (19) and recorded.

Surgery and postoperative chemotherapy. The surgical procedure applied was either total gastrectomy for proximal tumors or subtotal gastrectomy when the primary tumor was located distally in the 
Table II. Toxicity associated with intraperitoneal and systemic chemotherapy $(n=17)$.

\begin{tabular}{|c|c|c|c|c|c|c|c|c|c|c|}
\hline \multirow[b]{3}{*}{ Toxicity } & \multicolumn{10}{|c|}{ Grade* } \\
\hline & \multicolumn{5}{|c|}{ Intraperitoneal chemotherapy } & \multicolumn{5}{|c|}{ Systemic chemotherapy } \\
\hline & $1(\mathrm{n})$ & $2(n)$ & $3(n)$ & $4(n)$ & $3 / 4(\%)$ & $1(\mathrm{n})$ & $2(n)$ & $3(n)$ & $4(n)$ & $3 / 4(\%)$ \\
\hline \multicolumn{11}{|l|}{ Hematological } \\
\hline Leukopenia & 2 & 2 & 0 & 0 & 0 & 1 & 5 & 5 & 2 & 41 \\
\hline Neutropenia & 2 & 2 & 0 & 0 & 0 & 1 & 3 & 5 & 4 & 53 \\
\hline Thrombocytopenia & 0 & 0 & 0 & 0 & 0 & 6 & 1 & 2 & 0 & 12 \\
\hline Anemia & 4 & 2 & 0 & 0 & 0 & 2 & 7 & 5 & 0 & 29 \\
\hline AST elevation & 2 & 0 & 0 & 0 & 0 & 3 & 0 & 0 & 0 & 0 \\
\hline ALT elevation & 2 & 0 & 0 & 0 & 0 & 4 & 1 & 1 & 0 & 6 \\
\hline Bilirubin & 2 & 0 & 0 & 0 & 0 & 2 & 0 & 0 & 0 & 0 \\
\hline BUN & 0 & 0 & 0 & 0 & 0 & 2 & 0 & 0 & 0 & 0 \\
\hline Creatinine & 0 & 0 & 0 & 0 & 0 & 1 & 2 & 1 & 0 & 6 \\
\hline \multicolumn{11}{|l|}{ Gastrointestinal } \\
\hline Anorexia & 0 & 0 & 0 & 0 & 0 & 6 & 1 & 0 & 0 & 0 \\
\hline Nausea & 0 & 0 & 0 & 0 & 0 & 4 & 2 & 0 & 0 & 0 \\
\hline Vomiting & 0 & 0 & 0 & 0 & 0 & 0 & 0 & 0 & 0 & 0 \\
\hline Diarrhea & 0 & 0 & 0 & 0 & 0 & 2 & 1 & 2 & 0 & 12 \\
\hline Fatigue & 0 & 0 & 0 & 0 & 0 & 6 & 2 & 0 & 0 & 0 \\
\hline Rash & 0 & 0 & 0 & 0 & 0 & 0 & 0 & 1 & 0 & 6 \\
\hline Abdominal pain & 0 & 0 & 0 & 0 & 0 & 0 & 0 & 0 & 0 & 0 \\
\hline Neurosensory & 0 & 0 & 0 & 0 & 0 & 0 & 0 & 0 & 0 & 0 \\
\hline
\end{tabular}

AST: Aspartate transaminase; ALT: alanine transaminase; BUN: blood urea nitrogen. *NCI-CTCAE version 4.0 (19).

stomach, with a 5-cm 'safe' margin in both. In all cases, en bloc D2 lymph node dissection was performed according to the Japanese Gastric Cancer Association guidelines (20). Intraoperative and postoperative complications were reported according to the Clavien-Dindo classification (21).

No earlier than 1 week after the operation, postoperative chemotherapy was commenced. Initially, the protocol was one course of weekly paclitaxel $\left(80 \mathrm{mg} / \mathrm{m}^{2}\right.$ i.v. $)$ on days 1,8 , and 15 , followed by 7 days of rest, followed by $\mathrm{S}-1\left(80 \mathrm{mg} / \mathrm{m}^{2} /\right.$ day $i . v$. $)$ for 28 days, followed by 14 days of rest. This protocol was followed for more than 1 year or until recurrence was confirmed (17).

Statistical analyses. Because no standard treatment for GC with PM has been established, a threshold for the 1-year OS rate of $54.0 \%$ was adopted based on the results of phase III clinical trial in non-resectable or recurrent GC after systemic administration of $\mathrm{S}-1$ and cisplatin (12). The expected 1-year OS rate was $65.7 \%$, based on our previously reported phase II study (11). We assumed a null hypothesis of $54.0 \%$ and an alternative hypothesis of $65.7 \%$, with a one-sided, type I error of 0.05 and power of 0.8 , an accrual time of 2 years, and follow-up of 1 year after closure of the recruitment phase. The planned sample size was 27 , according to the Southwest Oncology Group One-Arm Survival program (22).

Survival analyses were performed using the Kaplan-Meier method. OS was calculated from the date of the first staging laparoscopy to death from any cause or the last follow-up. All statistical analyses were performed using SAS software (version 9.4; SAS Institute, Cary, NC, USA).

\section{Results}

Patient characteristics and tumor response. A total of 17 patients (10 men, seven women; mean age=60 years, range=39-71 years) who had GC with PM were enrolled in this study between September 2010 and August 2015. Patient and tumor characteristics are shown in Table I. Macroscopically, infiltrating-type tumors (types 3 and 4) (20) accounted for $16 / 17$ of the cases (94\%). Histopathologically, undifferentiated tumors (including poorly differentiated and signet ring cell carcinoma) dominated $(11 / 16,65 \%)$. Tumor $\mathrm{T}$ status was estimated to be T4a or T4b. The PM lesions were located mainly on the diaphragm, falciform ligament, and peritoneum.

Among these 17 patients, five had target lesions. Four $(80 \%)$ of these patients showed a partial response, and one (20\%) had stable disease. Among the other 12 patients who did not have a measurable target lesion, eight $(66 \%)$ had a $30 \%$ decrease in wall thickness. Finally, two patients showed no change, and two showed increased disease progress (Table I). Hence, according to our evaluation of the antitumor effect, 12 out of $17(70.5 \%)$ patients were responders to the chemotherapeutic regimen.

Toxicity of intraperitoneal and systemic chemotherapy. Hematological and non-hematological toxic reactions are 
Table III. Surgical findings and postoperative complications $(n=11)$.

\begin{tabular}{lc}
\hline Finding & Number \\
\hline PM/peritoneal cytology status & \\
No PM/CY0 & 10 \\
No PM/CY1 & 1 \\
Type of surgery & \\
Total gastrectomy & 8 \\
Distal gastrectomy & 3 \\
Lymph node dissection & \\
D2 & 11 \\
Curability & \\
R0 & 10 \\
R1 & 1 \\
Postoperative complications & \\
Leakage & 0 \\
Postoperative bleeding & 0 \\
Pancreatic fistula & 0 \\
Intra-abdominal abscess & $1(\mathrm{Gr} 2)$ \\
Pneumonia & 0 \\
Wound infection & $1(\mathrm{Gr} 0)$ \\
Stomal stenosis & 0 \\
\hline
\end{tabular}

Gr: Toxicity grade according to the Clavien-Dindo classification (21), PM: peritoneal metastasis, CY0: peritoneal cytology negative for carcinoma cells, CY1: peritoneal cytology positive for carcinoma cells.

shown in Table II. No patients experienced abdominal pain related to IPC. The incidence of adverse events associated with IPC was extremely low, with no patients developing grade 3-4 adverse reactions.

Regarding the systemic chemotherapy regimen, the scheduled eight courses of PCS chemotherapy were performed in only 12 patients. The median number of systemic chemotherapy cycles administered per patient was seven (range $=2-8$ ). One patient underwent only two courses because progressive disease was found during the evaluation of chemotherapeutic efficacy. One patient underwent only five courses because of a grade 3 allergy to cisplatin.

During the systemic chemotherapy regimen, a grade 4 toxic reaction, manifesting as neutropenia and leukopenia, was observed in four and two patients, respectively. The most frequent grade $3 / 4$ toxic effects included leukopenia $(41 \%)$, neutropenia $(53 \%)$, thrombocytopenia $(12 \%)$, anemia (29\%), ALT elevation (6\%), and creatinine elevation (6\%). The non-hematological toxicities were generally mild and manageable except in one patient with a grade 3 rash during cisplatin administration and two patients with grade 3 diarrhea. Fatigue was the most common non-hematological toxicity (Table II). There were no treatment-related deaths with i.p. or systemic chemotherapy.

Surgical findings and postoperative complications. In all, two out of the 17 patients showed clinical disease
Table IV. Pathological findings $(n=11)$.

\begin{tabular}{lc}
\hline Finding & Number \\
\hline Depth of tumor invasion & \\
T0 & 1 \\
T1a & 1 \\
T1b & 1 \\
T2 & 1 \\
T3 & 6 \\
T4a & 1 \\
T4b & 0 \\
Lymph node metastasis & \\
N0 & 5 \\
N1 & 1 \\
N2 & 3 \\
N3a & 1 \\
N3b & 1 \\
JGCA stage & \\
01 & \\
IA & 2 \\
IB & \\
IIA & \\
IIB & 0 \\
IIIA & 2 \\
IIIB & 2 \\
IIIC & 2 \\
IV & 1 \\
JGCA histological response & \\
Grade 0 & 0 \\
Grade 1a & 1 \\
Grade 1b & \\
Grade 2 & \\
Grade 3 & 3 \\
\hline & 5 \\
\hline
\end{tabular}

JGCA: Japanese Gastric Cancer Association (20).

progression but the other 15 underwent second-look laparoscopy. Among them, PM disappearance was confirmed in 11, with the other four still showing evidence of PM. The conversion rate from PM to no PM was therefore $64.7 \%$ (11/17). Among the 11 with successful chemotherapy, gastrectomy with D2 dissection was undertaken in 10 patients with no PM and peritoneal cytology negative for carcinoma cells (CY0) and one patient with no PM and peritoneal cytology positive for carcinoma cells (CY1). Other surgical findings are shown in Table III. Among the 11 patients who underwent surgery, postoperative complications occurred in two $(18.2 \%)$. There were no surgery-related deaths.

Postoperative chemotherapy. Overall, 10 out of the 11 patients who underwent gastrectomy with D2 dissection were postoperatively given weekly paclitaxel in one course and S1 adjuvant chemotherapy in another. The other patient (no PM, CY0) was not given any postoperative adjuvant 


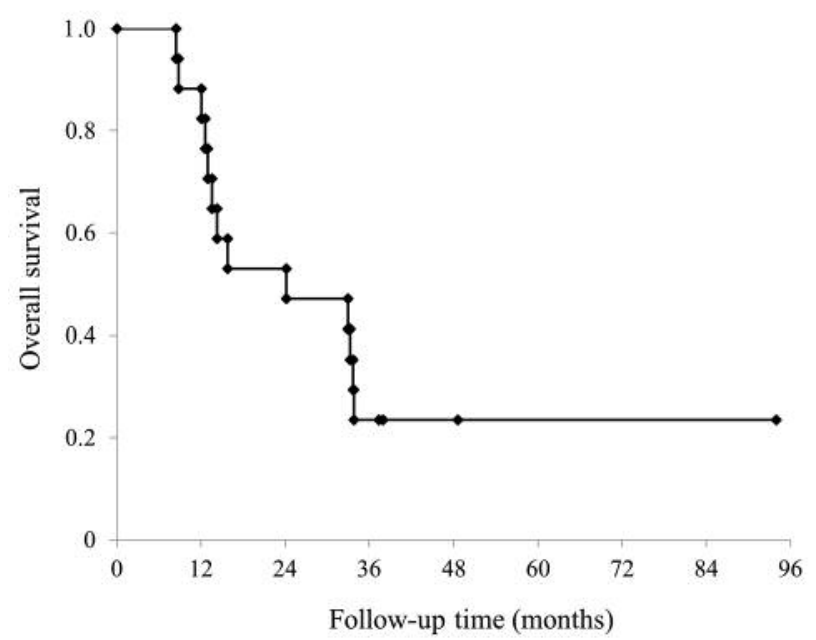

Figure 1. Kaplan-Meier estimates of overall survival of the 17 study patients.

chemotherapy because they were unwilling to undergo further treatment. The adverse events of postoperative chemotherapy were relatively mild, and there were no grade 4 toxic effects throughout the treatment period.

Pathological findings. The classifications and histological responses to chemotherapy were assessed according to the criteria of the Japanese Classification of Gastric Carcinoma, Third English Edition (20). The histological response of the primary lesion according to those criteria were grade $1 \mathrm{a}$ in five patients, grade $1 \mathrm{~b}$ in three, grade 2 in two, and grade 3 in one. The pathological response rate $(>1 \mathrm{~b})$ was $6 / 11$ $(54.5 \%)$. Other details of the final postoperative pathological findings for the 11 patients who underwent surgery are shown in Table IV.

Survival. At the time of this analysis (June 2018), 13 out of the 17 patients ( $76 \%$ ) had died, and the remaining four were alive without recurrence.

The 1-year OS rate was $82.4 \%$, with the lower limit of the $90 \%$ confidence interval (CI) at $60.5 \%$, which is higher than the threshold of $54.0 \%$. Furthermore, the $95 \%$ CI was $54.7-$ $93.9 \%$, with the lower limit still higher than the $54.0 \%$ threshold. The respective 2-and 5-year OS rates were $47.1 \%$ (95\% CI=23.0-68.0\%) and 23.5\% (95\% CI=7.3-44.9\%). The median survival time (MST) was 23.9 months (95\% $\mathrm{CI}=12.5-33.3$ months) (Figure 1). For the 11 patients who underwent R0 or R1 curative resection, the 1-, 2-, and 3-year OS rates were $90.9 \%$ (95\% CI=50.1-98.7\%), 63.6\% (29.7$84.5 \%$ ), and $36.4 \%$ (11.1-62.7\%), respectively, and the MST was 32.8 months ( $95 \% \mathrm{CI}=12.8$ months to not yet reached).

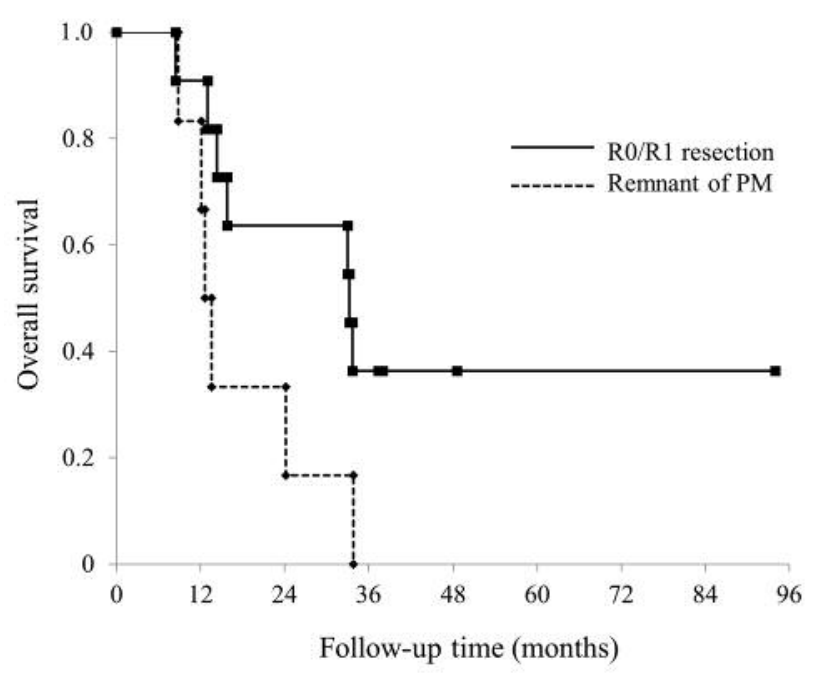

Figure 2. Kaplan-Meier estimates of the overall survival of the RO/RI resection group and the group with remnant of peritoneal metastasis (PM).

For the six patients who still had PM, the 1-and 2-year OS rates were $66.7 \%$ (95\% $\mathrm{CI}=19.5-90.4 \%)$ and $16.7 \%(95 \%$ $\mathrm{CI}=0.1-51.7 \%$ ), respectively, and the MST was 12.9 months (95\% CI=8.7-33.3 months).

None of the patients who were given chemotherapy only survived beyond 3 years (Figure 2). Among the seven patients who experienced recurrence after D2 gastrectomy, the peritoneum was the most frequent site of recurrence (in five out of the seven patients, $71 \%$ ). One patient each had a recurrence in the liver and lung, respectively.

\section{Discussion}

We conducted a prospective phase II study to evaluate the feasibility and efficacy of i.p. paclitaxel and systemic PCS chemotherapy plus subsequent surgery in patients with PM of GC. The study originally planned to include 27 patients. However, patient enrollment had been delayed and finally terminated before the projected number of patients was achieved because of the eligibility criteria. Most patients with PM tend to have relatively poor performance status and impaired organ function. In addition, during the research period, there were new laws and regulations in Japan stating that we could no longer administer paclitaxel intraperitoneally. Therefore, our phase II study completed registration with only 17 patients.

After i.p. paclitaxel and systemic PCS chemotherapy, 12 $(70.5 \%)$ out of the 17 patients were diagnosed as responders. Macroscopically, $11(64.8 \%)$ out of the 17 patients had negative results for PM presence. Additionally, our new combination regimen led to a 1 -year OS rate of $82.4 \%$, and the MST of all patients was 23.9 months. No patients 
developed grade 3-4 adverse reactions after i.p. paclitaxel chemotherapy in this study. In contrast, systemic PCS chemotherapy induced neutropenia and leukopenia as the main toxicities. The incidence of such hematological adverse events in our study was similar to those in a previous phase II study of a combination of PCS triplet chemotherapy in patients with non-resectable or metastatic GC (13-15). There were no unexpected serious adverse events or deaths related to the chemotherapy.

We therefore concluded that our i.p. paclitaxel regimen is effective, safe, and less toxic than other regimens. These findings might be explained by the pharmacokinetics of i.p. paclitaxel, with its low concentration in the systemic circulation compared with a higher concentration in the abdominal cavity (10).

We observed postoperative complications in only two out of the 11 patients (18.2\%) who underwent D2 gastrectomy, which is infrequent compared to the results in previous reports of surgery after neoadjuvant chemotherapy $(23,24)$. No surgery-related mortality was observed.

Previously, we reported the feasibility and efficacy of i.p. paclitaxel followed by five cycles of intravenous doublet chemotherapy of paclitaxel and S-1, with surgery for patients with PM of GC (11). Among 35 patients in that study, 22 (62.8\%) showed no macroscopic PM. The 1-year OS rate was $68.6 \%$, and MST for patients overall was 21.3 months. Compared to these previous data, the i.p. paclitaxel and PCS triplet regimen might be more effective than our previous regimen.

Regarding the number of doses of IPC, Yamaguchi et al. designed a phase II trial that combined repeated i.p. paclitaxel with $S-1$ plus intravenous paclitaxel in 35 patients who had GC with PM. Gastrectomy was performed in 21 patients after chemotherapy. Their 1-year OS rate and MST were $77.1 \%$ and 17.6 months, respectively (25). Our regimen comprises a single i.p. administration of paclitaxel with 1year OS rate and MST in our study slightly better than those reported by Yamaguchi et al.

As for the safety of the two techniques, Yamaguchi et al. reported complications related to the peritoneal access device, including obstruction of the i.p. catheter and infection of the access port (25). We experienced none of these complications because we did not use the access port. These results suggest that, generally, our regimen might be more advantageous.

Fujiwara et al. evaluated the efficacy of i.p. docetaxel combined with S-1 in 18 patients who had GC with PM. Gastrectomy was then performed in 16 out of the 18 patients. The 1-year OS rate and MST were $76 \%$ and 24.6 months, respectively (26).

As docetaxel is known to exhibit kinetics after i.p. administration similar to those of paclitaxel, these results suggest that it is another promising agent for IPC.
The results of our current study indicate that a single i.p. administration of paclitaxel and intravenous PCS chemotherapy are feasible and potentially effective for GC patients with PM. This study had a single-arm, phase II design, however, further clinical trials are warranted to examine the clinical significance of this regimen.

Regarding i.p. administration of paclitaxel to patients with PM of GC, we are in the process of making an application based on public knowledge to the Ministry of Health, Labor, and Welfare of Japan. An "application based on public knowledge" is a marketing authorization filing that seeks additional approval for another indication (i.e. the i.p. administration of paclitaxel) for use of a currently approved drug.

\section{Conclusion}

In conclusion, our novel multimodal treatment for GC with PM is feasible and effective. This regimen should be evaluated further in a randomized phase III trial.

\section{Conflicts of Interest}

None of the Authors have any conflicts of interest to declare in regard to this study.

\section{References}

1 Siewert JR, Bottcher K, Roder JD, Busch R, Hermanek P and Meyer HJ: Prognostic relevance of systematic lymph node dissection in gastric carcinoma. German Gastric Carcinoma Study Group. Br J Surg 80: 1015-1018, 1993.

2 Allum WH, Powell DJ, McConkey CC and Fielding JW: Gastric cancer: A 25-year review. Br J Surg 76: 535-540, 1989.

3 Sugarbaker $\mathrm{PH}$ and Yonemura Y: Clinical pathway for the management of resectable gastric cancer with peritoneal seeding: Best palliation with a ray of hope for cure. Oncology 58: 96-107, 2000.

4 Fujimoto S, Takahashi M, Kobayashi K, Kure M, Mutou T, Masaoka $\mathrm{H}$ and Ohkubo $\mathrm{H}$ : Relation between clinical and histologic outcome of intraperitoneal hyperthermic perfusion for patients with gastric cancer and peritoneal metastasis. Oncology 50: 338-343, 1993.

5 Ajani JA: Standard chemotherapy for gastric carcinoma: Is it a myth? J Clin Oncol 18: 4001-4003, 2000.

6 Kobayashi M, Sakamoto J, Namikawa T, Okamoto K, Okabayashi T, Ichikawa K and Araki K: Pharmacokinetic study of paclitaxel in malignant ascites from advanced gastric cancer patients. World J Gastroenterol 12: 1412-1415, 2006.

7 Yamada Y, Shirao K, Ohtsu A, Boku N, Hyodo I, Saitoh H, Miyata Y and Taguchi T: Phase II trial of paclitaxel by threehour infusion for advanced gastric cancer with short premedication for prophylaxis against paclitaxel-associated hypersensitivity reactions. Ann Oncol 12: 1133-1137, 2001.

8 Narahara H, Fujitani K, Takiuchi H, Sugimoto N, Inoue K, Uedo $\mathrm{N}$, Tsukuma H, Tsujinaka T, Furukawa $\mathrm{H}$ and Taguchi T: Phase II study of a combination of S-1 and paclitaxel in patients with unresectable or metastatic gastric cancer. Oncology 74: 37-41, 2008. 
9 Mochiki E, Ohno T, Kamiyama Y, Aihara R, Haga N, Ojima H, Nakamura J, Ohsawa H, Nakabayashi T, Takeuchi K, Asao T and Kuwano H: Phase I/II study of S-1 combined with paclitaxel in patients with unresectable and/or recurrent advanced gastric cancer. Br J Cancer 95: 1642-1647, 2006.

10 Imano M, Peng YF, Itoh T, Nishikawa M, Satou T, Yasyda A, Inoue $\mathrm{K}$, Kato $\mathrm{H}$, Shinkai M, Tsubaki M, Yasuda T, Imamoto $\mathrm{H}$, Nishida S, Furukawa H, Takeyama Y, Okuno K and Shiozaki H: A preliminary study of single intraperitoneal administration of paclitaxel followed by sequential systemic chemotherapy with S-1 plus paclitaxel for advanced gastric cancer with peritoneal metastasis. Anticancer Res 32: 4071-4076, 2012.

11 Imano M, Yasuda A, Itho T, Satou T, Peng YF, Kato H, Shinkai M, Tsubaki M, Chiba Y, Yasuda T, Imamoto H, Nishida S, Takeyama Y, Okuno K, Furukawa H and Shiozaki H: Phase II study of single intraperitoneal chemotherapy followed by systemic chemotherapy for gastric cancer with peritoneal metastasis. J Gastrointestinal Surg 16: 2190-2196, 2012.

12 Koizumi W, Narahara H, Hara T, Takagane A. Akiya T, Takagi M, Miyashita K, Nishizaki T, Kobayashi O, Takiyama W, Toh Y, Nagaie T, Takagi S, Yamamura Y, Yanaoka K, Orita H and Takeuchi M: S-1 plus cisplatin versus S-1 alone for first-line treatment of advanced gastric cancer (SPIRITS trial): A phase III trial. Lancet Oncol 9: 215-221, 2008.

13 Iwase H, Shimada M, Tsuzuki T, Ina K, Sugihara M, Haruta J, Shinoda M, Kumada T and Goto H: A Phase II multi-center study of triple therapy with paclitaxel, S-1 and cisplatin in patients with advanced gastric cancer. Oncology 80: 76-83, 2011.

14 Fujitani K, Hasegawa H, Hirao M, Kurokawa Y and Tsujinaka T: Feasibility study of triplet combination chemotherapy of paclitaxel, cisplatin and S-1 for advanced gastric cancer. Anticancer Res 31: 3085-3091, 2011.

15 Kim JY, Do TR, Park KU, Kim JG, Chae YS, Kim MK, Lee KH, Ryoo HM, Bae SH, Baek JH and Song HS: Multicenter phase II trial of S-1, paclitaxel and cisplatin triplet combination chemotherapy in patients with advanced gastric cancer. Cancer Chemother Phamacol 67: 527-532, 2011.

16 Williams JR: The Declaration of Helsinki and public health. Bull World Health Organ 86(8): 650-652, 2008.

17 Tsuburaya A, Sakamoto J, Morita S, Kodera Y, Kobayashi M, Miyashita Y and Macdonald JS: A randomized phase III trial of post-operative adjuvant oral fluoropyrimidine versus sequential paclitaxel/oral fluoropyrimidine: and UFT versus $\mathrm{S} 1$ for T3/T4 gastric cartinoma: The Stomach Cancer Adjuvant Multiinstitutional Trial Group (Samit) Trial Jpn J Clin Oncol 35: 672675,2005
18 Eisenhauer EA, Therasse P, Bogaerts J, Schwartz LH, Sargent D, Ford R, Dancey J, Arbuck S, Gwyther S, Mooney M, Rubinstein L, Shankar L, Dodd L, Kaplan R, Lacombe D and Verweij J: New response evaluation criteria in solid tumours: Revised RECIST guideline (version 1.1). Eur J Cancer 45(2): 228-247, 2009.

19 U.S. Department of Health and Human Services, National Cancer Institute: Common Terminology Criteria for Adverse Events (CTCAE) Version 4.0, 2009.

20 Japanese Gastric Cancer Association. Japanese Classification of Gastric Carcinoma: 3rd English edition. Gastric Cancer 14: 101112, 2011

21 Dindo D, Demartines N and Clavien PA: Classification of surgical complications: A new proposal with evaluation in a cohort of 6336 patients and results of a survey. Ann Surg 240: 205-213, 2004.

22 Doroshow JH, McCoy S, Macdonald JS, Issell BF, Patel T, Cobb PW, Yost KJ and Abbruzzese JL: Phase II trial of PN401, 5-FU, and leucovorin in unresectable or metastatic adenocarcinoma of the stomach: a Southwest Oncology Group study. Invest New Drugs 24(6): 537-542, 2006.

23 Yoshikawa T, Sasako M and Yamamoto S: Phase II study of neoadjuvant chemotherapy and extended surgery for locally advanced gastric cancer. Br J Surg 96: 1015-1022, 2009.

24 Okabe H, Ueda S and Obama K: Induction chemotherapy with S-1 plus cisplatin followed by surgery for treatment of gastric cancer with peritoneal dissemination. Ann Surg Oncol 16: 32273236, 2009 .

25 Yamaguchi H, Kitayama J, Ishigami H, Emoto S, Yamashita H and Watanabe T: A phase 2 trial of intravenous and intraperitoneal paclitaxel combined with S-1 for treatment of gastric cancer with macroscopic peritoneal metastasis. Cancer 119: 3354-3358, 2013.

26 Fujiwara Y, Takiguchi S, Nakajima K, Miyata H, Yamasaki M, Kurokawa Y, Mori M and Doki Y: Intraperitoneal docetaxel combined with S-1 for advanced gastric cancer with peritoneal dissemination. J Surg Oncol 105: 38-42, 2012.
Received September 3, 2018

Revised September 19, 2018

Accepted September 21, 2018 